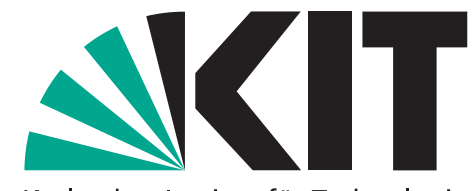

Karlsruher Institut für Technologie

\title{
Negative recency, randomization device choice, and reduction of compound lotteries
}

by Kim Kaivanto and Eike B. Kroll

No. 22 | APRIL 2011

\section{WORKING PAPER SERIES IN ECONOMICS}

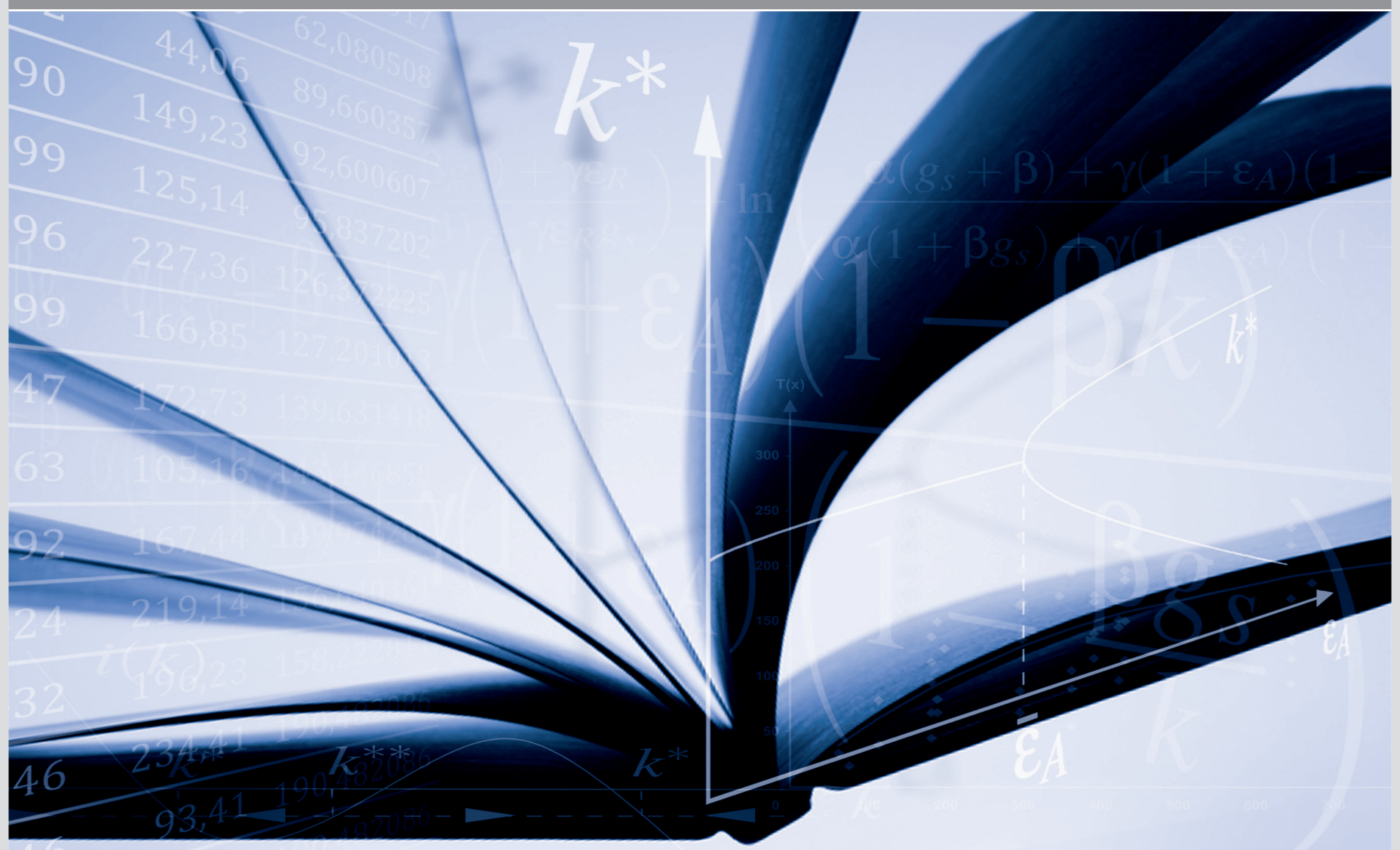




\section{Impressum}

Karlsruher Institut für Technologie (KIT)

Fakultät für Wirtschaftswissenschaften

Institut für Wirtschaftspolitik und Wirtschaftsforschung (IWW)

Institut für Wirtschaftstheorie und Statistik (ETS)

Schlossbezirk 12

76131 Karlsruhe

KIT - Universität des Landes Baden-Württemberg und nationales Forschungszentrum in der Helmholtz-Gemeinschaft

Working Paper Series in Economics

No. 22, April 2011

ISSN 2190-9806

econpapers.wiwi.kit.edu 


\title{
Negative Recency, Randomization Device Choice,
}

\section{And Reduction of Compound LotTeries*}

\author{
Kim Kaivanto ${ }^{\dagger}$ \\ Lancaster University, Lancaster LA1 4YX, UK \\ Eike B. Kroll \\ Karlsruhe Institute of Technology, Karlsruhe 76128, Germany \\ this version: April 14, 2011
}

\begin{abstract}
We report an experiment in which subjects are not indifferent between real-money lotteries implemented with randomization devices that are equivalent under the Reduction Axiom. Instead choice behavior is consistent with subjective distortion of conditional probability, and this persists in treatment conditions that control for (i) computational limitations and (ii) possible confounding by ratio bias.
\end{abstract}

Keywords: reduction of compound lotteries, negative recency effect, gambler's fallacy, law of small numbers, randomization devices, instruments and materials, design of experiments, St. Petersburg paradox JEL classification: D81, C91

${ }^{*}$ Copyright (C) 2011 Kim Kaivanto and Eike Kroll

${ }^{\dagger}$ tel +44(0)1524594030; fax +44(0)1524594244; e-mail k.kaivanto@lancaster.ac.uk 


\section{Introduction}

What specific factors lead to violations of the Reduction of Compound Lotteries Axiom? The question is consequential for Expected Utility Theory's (EUT's) domain of descriptive validity. It is also consequential for the design and interpretation of experiments. To date this question has not been examined in a systematic fashion within the economics literature.

This paper presents the results of an experiment designed to determine whether the negative recency effect - i.e. a subjective distortion of conditional probability - explains subjects' choices between onestage and two-stage variants of a real-money lottery. The overwhelming majority of subjects (80\%) choose the one-stage variant of the lottery. The direction and strength of this effect survives (i) being explicitly informed that the two lotteries are probabilistically equivalent, ruling out a miscalculation errors explanation (77\%), and (ii) controlling for the size of the sample space, which rules out a ratio bias explantion (80\%). This choice pattern is consistent with negative recency and inconsistent with the Reduction Axiom, which in contrast requires indifference between the two variants of the lottery.

\section{The Reduction Axiom}

The EU representation of preferences is underpinned by the Reduction of Compound Lotteries Axiom. In von Neumann and Morgenstern (1947) it appears as Axiom 3:C:b (p. 26). In Samuelson (1952) it appears in the definition of the objects of choice as being 'simple' lotteries, where any compound lottery is reduced to a "simple lottery ticket whose prizes are all the possible prizes of the compound lottery ticket, each evaluated with the compound probabilities that the classical algebra of probability defines. ...only algebra, not human behavior, is involved in this definition" (p. 671). The axiom is a necessary underpinning of EU (Halevy, 2007). ${ }^{1}$

Under the Reduction Axiom a decision maker is indifferent between a two-stage compound lottery and its 'collapsed', one-stage, probabilistically equivalent simple lottery. The Reduction Axiom limits the scope of applicability of EU to preferences that obey this property, on the one and, and is a defining characteristic of normative rationality, on the other.

The Reduction Axiom has become the focus of experimental investigation in connection with the Allais and Ellsberg paradoxes. EU-violating Allais-type choice patterns have been attributed to the joint violation of the Independence and Reduction Axioms (Conlisk, 1989; Carlin, 1992). And choice patterns in the Ellsberg setup, which are inconsistent with Subjective Expected Utility, have also been linked to violations of the Reduction Axiom (Bernasconi and Loomes, 1992; Halevy, 2007). This literature has concentrated primarily on establishing the fact of Reduction Axiom violations rather than testing for their

\footnotetext{
${ }^{1}$ See e.g. Segal (1990, Theorem 3); he also shows what combination of properties needs to be assumed if one is to forgo the Reduction Axiom: the combination of (i) one-stage strict stochastic dominance, (ii) compound independence, (iii) time neutrality and (iv) strong compound dominance.
} 
causes. The exception in this domain is contained in the work of Daniel Kahneman and Amos Tversky, who explain violations of the Reduction Axiom through the isolation, certainty and pseudo-certainty effects. ${ }^{2}$ Heretofore the question of whether Reduction Axiom violations can arise in other ways has not been taken up in the economics literature.

\section{The negative recency effect}

The strong prediction that negative recency holds for particular types of two-stage lotteries provides both the impetus for and the central hypothesis of the present experiment.

Negative recency is one aspect of the 'law of small numbers', which was introduced into the economics literature by Rabin (2002). ${ }^{3}$ The extensive experimental literature on the negative recency effect is summarized in Bar-Hillel and Wagenaar (1991). The effect, which gives rise to the gambler's fallacy, continues to be borne out in experimental economics investigations in the lab (see Asparouhava et al., 2009) as well as in the field, e.g. in the Maryland lottery's "Pick 3" numbers game (Clotfelter and Cook, 1993), in the New Jersey lottery's (pari-mutuel) three-digit numbers game (Terrell, 1994), and in the Kansas City greyhound races (Terrell, 1998).

Under the law of small numbers, people misjudge and "exaggerate how likely it is that a small sample resembles the parent population from which it is drawn" (Rabin, 2002, p. 775). As an empirical regularity, people erroneously expect (a) the frequency distributions of small samples to closely approximate the limiting frequency distributions, and (b) the 'irregularity' of small samples to be greater than that typically generated by fair, memoryless randomization devices. It is the latter, negatively distorted conditional subjective probability belief, that constitutes 'negative recency'. Under negative recency, when a memoryless randomization device generates a particular outcome $\omega_{i} \in\left\{\omega_{1}, \omega_{2}, \ldots, \omega_{n}\right\}$ with objective probability $\frac{1}{n}$, the subjective probability of a second draw yielding that same outcome $\omega_{i}$ is less than $\frac{1}{n}$.

The negative recency effect holds clear implications for preferences between one- and two-stage variants of a lottery: for lotteries with objectively identical win probabilities, subjects whose perception of randomness is characterized by negative recency will prefer the one-stage lottery to its two-stage variant. In other words, the Reduction Axiom's requirement of indifference between one- and two-stage variants is predicted to be violated under negative recency.

\section{Experiment}

This experiment is designed to test whether the negative recency effect causes the Reduction Axiom to be violated. For implementing the one- and two-stage lotteries, simple and familiar randomization devices

\footnotetext{
${ }^{2}$ See Kahneman and Tversky (1979) and Tversky and Kahneman (1981). Keller (1985) found that the relative strength of the certainty and pseudo-certainty effects is modulated by the form of the problem presentation.

${ }^{3}$ In the context of binary sequences, the negative recency effect is known as 'alternation bias'.
} 
are methodologically preferable, and in this experiment we use dice. ${ }^{4}$

The experiment was conducted in pen and paper format at the experimental laboratory of the Karlsruhe Institute of Technology (KIT). Ninety students from different fields of study were recruited and assigned to the three treatment conditions using ORSEE (Greiner, 2004). Each treatment was implemented across three sessions of 10 subjects each lasting 30 minutes on average. Subjects ranged in age from 22 to 28 , with an average of 24.1 years. $69 \%$ of the subjects were male. At the time of participating in the study, all subjects were enrolled in an engineering or computer science degree program at KIT, and had already completed at least one course in mathematics or statistics as part of their degree program.

At the beginning of each session, subjects were given written instructions describing the lottery and their choice alternatives. After subjects had time to read the instructions, the experimenter demonstrated the randomization devices to be used in the experimental procedure. Before distribution of answer sheets, subjects were given the opportunity to seek clarification on any aspect of the experiment. Subjects wishing to ask a question were individually led outside the laboratory room, where neither the question nor the answer could be heard by the other subjects. After all participants completed their respective answer sheets, the experimenter proceeded to implement the lottery for each subject using his/her chosen randomization device. Standard laboratory protocols to minimize the risk of experimenter demand effects were followed.

\subsection{Base treatment condition}

Participating subjects are individually presented with a real-money lottery in which they stand a $10 \%$ chance of winning 100 Euro. ${ }^{5}$ The lottery is implemented by a random draw of one integer between 1 and 100, where the monetary prize is won if the integer is composed of two identical digits $(11,22,33$, ..., 100). In the event that non-identical digits are drawn, the subject's payoff is zero. Each subject is offered a choice between two objectively interchangeable randomization devices. Option 1 is one roll of a 100-sided die. Option 2 is two rolls of a 10-sided die.

Regardless of which device is chosen, the objective probability of winning 100 Euro is $\frac{1}{10}$. The null hypothesis to be tested is that subjects display no preference bias toward either device, i.e. that the proportion $\hat{p}$ selecting the 100 -sided die is $\frac{1}{2}$. The finding of a systematic bias toward either device is not consistent with choice based jointly and solely on normative (rational) decision theory and probability theory. In EUT this joint requirement is formalized in the Reduction of Compound Lotteries Axiom. Rejection of the null hypothesis on a two-sided binomial test of $\hat{p}=0.5$ implies rejection of Reduction

\footnotetext{
${ }^{4}$ 10-sided (pentagonal trapezohedron) and 100-sided (Zocchihedron) dice, as used here, generate sample spaces that are familiar and convenient in the base 10 number system. The associated natural frequency representations of probability and multiplicative operations carried out on these fractions involve lower 'computation costs' than with alternatives such as 4-sided (tetrahedron) and 16-sided (octagonal dipyramid) dice.

${ }^{5}$ The laboratory sessions were conducted in February and March 2011. In February the average US-currency value of 100 Euro was $\$ 136.45$, while in March it was $\$ 139.90$.
} 
Axiom. $H_{0}: \hat{p}-0.5=0, H_{1}: \hat{p}-0.5 \neq 0$.

Rejection of this null hypothesis is also consistent with an alternative that maintains the Reduction Axiom as part of a joint hypothesis. Where subjects are indifferent between the two devices under the Reduction Axiom, nothing is lost if an additional cue or criterion tips choice toward one option or the other. Here the alternative hypothesis would be that subjects differentiate lexicographically between the devices when they are equally preferred under the Reduction Axiom.

We note that such a lexicographically augmented reduction axiom is not in keeping with the EUT of rational choice literature, in which the only attributes to be legitimately (rationally) consulted are the probabilities and payoffs of the reduced, single-stage, simple lottery. Furthermore, it is not in keeping with the principle of parsimony. ${ }^{6}$

Subjects whose perception of randomness is characterized by the law of small numbers - and in particular negative recency, under which conditional probability beliefs are distorted - may be expected to differentiate between the two randomization devices. Because the 100-sided die implements the draw in a single roll whereas the 10-sided die implements the draw in two sequential rolls, subjective distortions of conditional probability beliefs can affect the subjective evaluation of the latter (10-sided) device but not the former (100-sided) device. Specifically, as a result of negative recency the subjective (distorted) conditional probability - of the second roll of the 10-sided die landing on the same digit as on the first roll - is less than $\frac{1}{10}$. To the extent that subjects' perception of randomness embodies negative recency, ceteris paribus such subjects will display a preference for the 100-sided die. Thus the corresponding alternative hypothesis is directional $H_{1}^{\mathrm{nr}}: \hat{p}-0.5>0$.

\subsection{Zero computation cost treatment condition}

The cognitive resources of any one individual being finite, the fact that computation and deliberation is 'costly' in terms of time, attention and mental effort entails that when confronted with particular types of tasks, an individual may face a binding cognitive constraint which prevents full rationality (Pingle, 2010). As Simon (1955) observed, the rationality that a subject is capable of displaying is that which is "compatible with the access to information and computation that are actually possessed" (p. 99).

Two-stage compound lotteries, as opposed to one-stage simple lotteries, require attention, reasoning and probability calculation in order to be reduced into one-stage lotteries that are directly comparable with other one-stage lotteries.

Although a remote possibility considering the training of the subject pool, it is conceivable that some base case treatment condition subjects may not have premised their choice on a correct appreciation of the fact that both randomization devices yield a win with probability $\frac{1}{10}$. In other words, it is conceivable that 'deliberation' or 'computation' costs may prevent some subjects from making the choice they would

\footnotetext{
${ }^{6}$ One of the authors has suggested that this joint alternative be dubbed the lexicographic epicycle hypothesis.
} 
make in the absence of such costs (Carlin, 1992). ${ }^{7}$

In order to rule out this possibility, computation cost is reduced to zero in this treatment condition through the inclusion of a paragraph that compares the randomization devices from the standpoint of objective probability theory. The paragraph explains and makes explicit the fact that both randomization devices offer the same $\frac{1}{10}$ probability of winning 100 Euro.

The null and alternative hypotheses for this treatment condition are the same as in the base case.

\subsection{Ratio bias control treatment condition}

10-sided and 100-sided dice differ in the size of the sample spaces they respectively define. Whereas these particular dice ensure familiarity and minimize computation costs, any systematic preference for the single-stage lottery implemented with a 100-sided die could be due to either (a) the negative recency effect or (b) an effect associated with the difference in sample spaces (10 vs. 100).

Indeed the logical possibility in (b) has a counterpart in the heuristics and biases literature: the ratio bias effect. Studies exemplified by Denes-Raj and Epstein (1994) and Pinto-Prades et al. (2006) find that in choices between lotteries having identical win probabilities, subjects choose the lottery representation with the greater number of potential successes, i.e. the representation with the larger sample space. ${ }^{8}$ This preference for the 'ratio of large numbers' appears to persist even when the objective probability of the large sample space option has a strictly inferior win probability, and even when subjects are explicitly informed of the objective probabilities (zero computation cost) (Denes-Raj and Epstein, 1994).

In order to rule out ratio bias as a driver of the observed choice pattern, the sample space sizes are equalized between the two devices in this control treatment condition. As before, option 1 is one roll of a 100-sided die; the probability of winning is 10-in-100. Option 2 is amended to two rolls of a 100-sided die, where attention is restricted to the final digit; whatever final digit is realized on the first roll, there are 10-in-100 chances of realizing the same final digit on the second roll.

The null and alternative hypotheses for this treatment condition are the same as in the base case.

\section{$5 \quad$ Results}

Of the 90 subjects who participated in this real-money lottery choice experiment, 6 won the prize of 100 Euro. The data reveal a strong and consistent preference for the 100-sided die (the single-stage lottery). Formal testing employs the binomial test. The Reduction Axiom is rejected in the base case treatment condition as well as in the control conditions with two-sided $p<0.01$. Under the one-sided test consistent with the alternative under the negative recency effect $H_{1}^{\mathrm{nr}}: \hat{p}-0.5>0$ the null hypothesis is rejected

\footnotetext{
${ }^{7}$ As Pingle (2010) emphasizes, it is an imperative of good methodological practice to not overlook the possibility that deliberation cost under scarcity of cognitive resources acts as a binding constraint on rationality.

${ }^{8}$ Small sample space option: a bowl containing 10 jelly beans, one of which is red. Large sample space option: a bowl containing 100 jelly beans, ten of which are red.
} 
Table 1: Device preference for implementation of 1-in-10 chance to win 100 Euro. Also reported are $p$-values of binomial test of $H_{0}: \hat{p}=0.5$.

\begin{tabular}{lccccc}
\hline Treatment condition & Simple lottery & Compound lottery & N & two-sided & one-sided \\
\hline Base case & 24 & 6 & 30 & 0.001431 & 0.000715 \\
Zero computation cost & 23 & 7 & 30 & 0.005223 & 0.002611 \\
Equal sample space & 24 & 6 & 30 & 0.001431 & 0.000715 \\
\hline
\end{tabular}

with $p<0.001$ in the base case and with $p<0.01$ in the control conditions. ${ }^{9}$ Thus the rejection of the Reduction Axiom is due neither to 'miscalculation' as the result of computation costs, nor to ratio bias reflecting the sample space size difference between the 10-sided die and the 100-sided die.

\section{Conclusion}

These results hold implications for EUT, experimental design, and the interpretation of experiments.

The Reduction Axiom of EUT is a condition that applies to the binary preference relation $(\succ, \sim, \prec)$ between simple and compound lotteries. It stipulates that decision makers will be indifferent between a compound lottery and its mathematically equivalent simple lottery. It does not state that agents are free to adduce additional criteria or cues upon which to base choice when, on the level of rational binary preferences, indifference holds. Choice is required to follow rational preferences.

The present experiment's results are consistent with the implications of the negative recency effect and inconsistent with the Reduction Axiom. Multi-stage lotteries such as the ones considered here, which repeatedly employ the same randomization device at each stage, are not descriptively interchangeable with their logically equivalent single-stage variants. This study shows that Reduction Axiom violations may not be restricted to the specific configurations of the Allais and Ellsberg Paradox experiments.

The implication for the design of experiments is that compound structures, whether part of a lottery's design or merely an incidental feature of the randomization device, can introduce biases into the resulting experimental data if subjects' perception of randomness is characterized by negative recency.

Equally, there are implications for the interpretation of experiments. Consider the St. Petersburg gamble ${ }^{10}$ which is clearly a multi-stage compound lottery. The Reduction Axiom is implicit in the standard statement of the expected value of the St. Petersburg gamble: $E\left(G_{\mathrm{StP}}\right)=\frac{1}{2} \cdot \$ 2+\left(\frac{1}{2}\right)^{2} \cdot \$ 2^{2}+\cdots$. In the context of coin tossing, empirical estimates of the first-order subjective distortion of conditional probability (i.e. negative recency) place the effect at $P(H \mid T)=.6$ (Bar-Hillel and Wagenaar, 1991). Accounting for first-order negative recency, the subjectively distorted expected value of the St. Petersburg gamble is not infinite but finite $E^{\mathrm{f}-\mathrm{o}}\left(G_{\mathrm{StP}}\right)=\frac{1}{2} \cdot 2+\sum_{n=2}^{\infty} \frac{1}{2} P(H \mid T) P(T \mid T)^{n-2} \cdot 2^{n}=1+0.3 \sum_{n=2}^{\infty} 0.4^{n-2} 2^{n}=7.0<\infty$.

\footnotetext{
${ }^{9}$ In the base case and the ratio bias control condition, the achieved statistical power is 0.939 (two sided) and 0.974 (one sided). In the computation cost control condition the achieved statistical power is 0.859 (two sided) and 0.930 (one sided). 10 typically either a hypothetical question or part of a thought experiment
} 


\section{References}

Asparouhava, E., Hertzel, M., Lemmon, M., 2009. Inference from streaks in random outcomes: Experimental evidence on beliefs in regime shifting and the law of small numbers, Management Science 55(1), $1766-1782$.

Bar-Hillel, M., Wagenaar, W.A., 1991. The perception of randomness, Advances in Applied Mathematics $12(4), 428-454$.

Bernasconi, M., Loomes, G., 1992. Failures of the reduction principle in an Ellsberg-type problem, Theory and Decision 32(1), 77-100.

Carlin, P.S., 1992. Violations of the reduction and independence axioms in Allais-type and common-ratio effect experiments, Journal of Economic Behavior and Organization 19(3), 213-235.

Clotfelter, C., Cook, P., 1993. The 'gambler's fallacy' in lottery play, Management Science, 39(12), 15211525.

Conlisk, J., 1989. Three variants of the Allais example, American Economic Review 79, 392-407.

Denes-Raj, V., Epstein, S., 1994. Conflict between intuitive and rational processing: When people behave against third better judgment, Journal of Personality and Social Psychology 66(5), 819-829.

Greiner, B., 2004. The online recruitment system ORSEE 2.0 - A guide for the organization of experiments in economics. Cologne: University of Cologne.

Halevy, Y., 2007. Ellsberg revisited: An experimental study, Econometrica 75(2), 503-536.

Kahneman, D., Tversky, A., 1979. Prospect theory: An analysis of decision under risk, Econometrica $47(2), 263-291$.

Keller, L.R., 1985. Testing of the 'reduction of compound alternatives' principle, Omega 13(4), 349-358.

von Neumann, J., Morgenstern, O., 1947. Theory of games and economic behavior, 2nd edition, (Princeton University Press, Princeton NJ).

Pingle, M., 2010. Looking under the hood: Exploring assumptions and finding behavioral economics, Journal of Economic Behavior and Organization 73(1), 730-76.

Pinto-Prades, J., Martinez-Perez, J., Abellan-Perpinan, J., (2006). The influence of the ratio bias phenomenon on the elicitation of health states utilities, Judgment and Decision Making 1(2), 118-133.

Rabin, M., 2002. Inference by believers in the law of small numbers, Quarterly Journal of Economics $117(3), 775-816$.

Samuelson, P.A., 1952. Probability, utility, and the independence axiom, Econometrica 20(4), 670-678.

Segal, U., 1990. Two-stage lotteries without the reduction axiom, Econometrica 58(2), 349-377.

Simon, H.A., 1955. A behavioral model of rational choice, Quarterly Journal of Economics 69(1), 99-118.

Terrell, D., 1994. A test of the gambler's fallacy: Evidence from pari-mutuel games, Journal of Risk and Uncertainty, 8(3), 309317.

Terrell, D., 1998. Biases in assessments of probabilities: New evidence from greyhound races, Journal of Risk and Uncertainty 17(2), 151-166.

Tversky, A., Kahneman, D., 1981. The framing of decision sand the psychology of choice, Science 211(4481), 453-458. 


\section{Working Paper Series in Economics}

recent issues

No. 22 Kim Kaivanto and Eike B. Kroll: Negative recency, randomization device choice, and reduction of compound lotteries, April 2011

No. 21 Antje Schimke and Thomas Brenner: Long-run factors of firm growth - a study of German firms, April 2011

No. 20 Aaron B. Scholz: Spatial network configurations of cargo airlines, April 2011

No. 19 Arne Beck: Public bus transport in Germany - a proposal to improve the current awarding system, April 2011

No.18 Nina Menz and Ingrid Ott: On the role of general purpose technologies within the Marshall-Jacobs controversy: the case of nanotechnologies, April 2011

No. 17 Berno Buechel: A note on Condorcet consistency and the median voter, April 2011

No. 16 Eike B. Kroll, Jörg Rieger and Bodo Vogt: How performance based payoffs influence estimates of complex information? An experimental study on quality and precision in estimation tasks, March 2011

No. 15 Siegfried K. Berninghaus, Thomas Neumann and Bodo Vogt: Learning in networks - an experimental study using stationary concepts, February 2011

No. 14 Marten Hillebrand: Governmental debt, interest policy, and tax stabilization in a stochastic OLG economy, January 2011

No. 13 Arne Beck and Matthias Walter: Tender prices in local bus transport in Germany - an application of alternative regression techniques, December 2010

No. 12 Eike B. Kroll, Holger Müller and Bodo Vogt: Experimental evidence of context-dependent preferences in risk-free settings, November 2010 